Disponível em

http://www.anpad.org.br/rac

RAC, Rio de Janeiro, v. 17, n. 4, art. 4, pp. 459-478, Jul./Ago. 2013

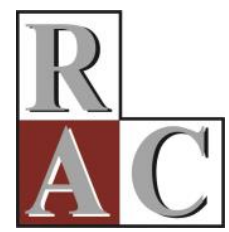

\title{
Inovação Global de Subsidiárias Estrangeiras Localizadas em Mercados Emergentes
}

\section{Global Innovation in Foreign Subsidiaries Located in Emerging Markets}

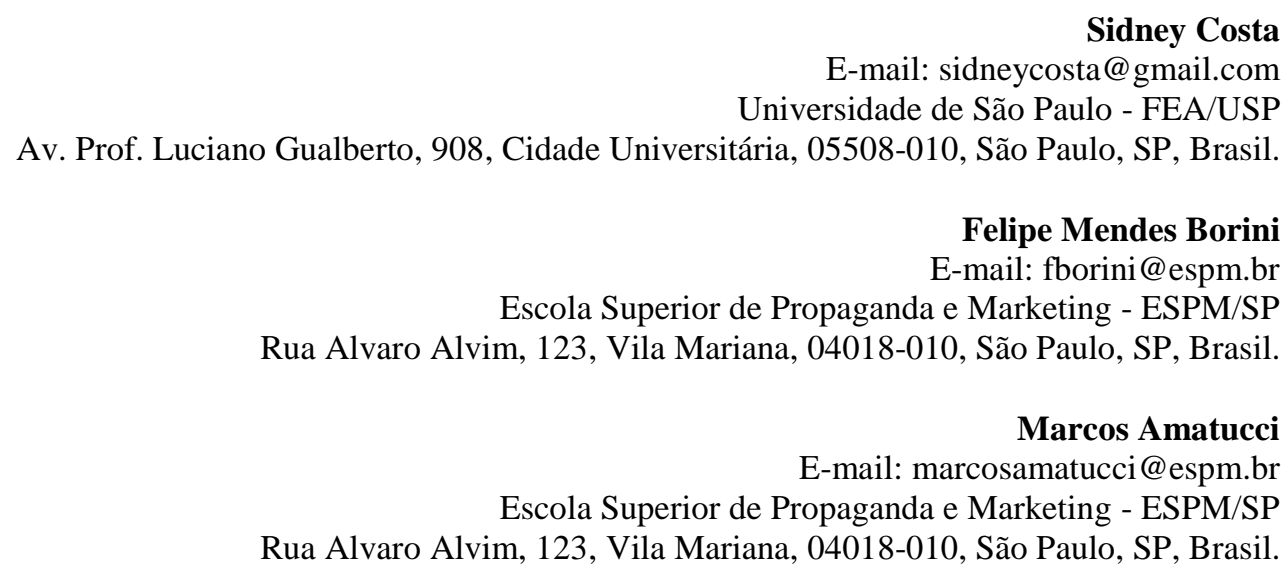

Artigo recebido em 14.11.2012. Última versão recebida em 08.04.2013. Aprovado em 20.04.2013. 


\title{
Resumo
}

O presente artigo tem como objetivo mostrar que a inovação global em subsidiárias estrangeiras instaladas em mercados emergentes depende dos contextos competitivos nacional e internacional. A dependência é direta no âmbito internacional e indireta no contexto competitivo nacional. Isso porque as subsidiárias precisam se inserir nas redes externas do país hospedeiro para se valer dos benefícios do contexto competitivo nacional. Nesse intuito, o artigo utiliza como objeto de investigação as subsidiárias estrangeiras instaladas no Brasil como representantes das subsidiárias hospedadas em mercados emergentes. Para tanto, faz uso de modelos de equações estruturais. O resultado sugere que as subsidiárias estrangeiras localizadas em mercados emergentes que praticam atividades de inovação global usam estratégias diferenciadas para acessar os recursos do ambiente externo. Para aproveitar os benefícios do contexto competitivo nacional do país hospedeiro, elas precisam se envolver em redes técnicas como meio de superar as adversidades do local e aprender a operar no mercado emergente. Por sua vez, as subsidiárias integradas em cadeias produtivas globais fazem uso direto do contexto competitivo internacional localizado em países desenvolvidos.

Palavras-chave: subsidiárias estrangeiras; estratégia de empresas multinacionais; inovação global.

\begin{abstract}
The purpose of this paper is to show that global innovation in foreign subsidiaries located in emerging markets depends directly on the international competitive context and indirectly on the national competitive context. This requires subsidiaries participate in networks external to the host country to benefit from a domestic competitive context. For this reason, the article uses foreign subsidiaries installed in Brazil as the object of research, as a representative sample of subsidiaries located in emerging markets. Structural equation modeling was applied to a survey conducted with subsidiaries in Brazil to test proposed hypotheses. The results show that foreign subsidiaries that carry out activities of global innovation while located in emerging markets use differentiated strategies to access resources in the external environment. In order to benefit from the host country's national competitive benefits, subsidiaries need to get involved in technical networks as a means of learning to operate in an emerging market and overcome domestic market challenges. Subsidiaries integrated in global supply chains make direct use of the international competitive contexts located in developed countries.
\end{abstract}

Key words: foreign subsidiaries; multinational strategies; innovation. 


\section{Introdução}

Um importante direcionador para a inovação global nas subsidiárias estrangeiras (Birkinshaw, 2001) é o contexto competitivo nacional, ou seja, a disponibilidade e a qualidade dos fornecedores de um determinado país, a demanda e o grau de exigência dos consumidores do local, assim como as condições para o estabelecimento da concorrência naquele mercado (Porter, 1990). Outros estudos debatem, também, a importância da inserção das subsidiárias nas redes externas como direcionadoras das inovações globais (Andersson, Forsgreen, \& Holm, 2002; Birkinshaw, Hood, \& Jonsson, 1998).

Contudo, esses estudos consideram o contexto competitivo e as redes externas como elementos que exercem impacto direto para a inovação global em subsidiárias. Por sua vez, o presente artigo questiona essa relação direta do contexto competitivo nacional na inovação global das subsidiárias localizadas em mercados emergentes. Nesses mercados, o contexto competitivo não é estruturado tal qual nos países desenvolvidos, o que exige que entrantes estrangeiros estabeleçam parcerias para suplantar os vazios institucionais (Khanna, Palepu, \& Bullock, 2010) e tenham acesso às inovações específicas dos mercados emergentes (Eyring, Johnson, \& Nair, 2011).

Como contraponto, o presente artigo defende que as subsidiárias estrangeiras em mercados emergentes não necessitam da inserção em redes quando fazem uso de contextos competitivos internacionais (Moon, Rugman, \& Verbeke, 1998; Rugman \& D'cruz, 1993) para suas inovações globais. Entende-se por contexto competitivo internacional a influência exercida por clientes, fornecedores e competidores localizados fora do mercado nacional (Moon et al., 1998; Rugman \& D’Cruz, 1993), mais especificamente neste artigo, em um país desenvolvido. A tese principal na qual se sustenta essa argumentação aponta que as subsidiárias estrangeiras em mercados emergentes - que fazem uso do contexto competitivo internacional - são empresas que também exercem o papel de um elo da cadeia produtiva internacional (Hiratuka, 2010). Elas se valem do contexto competitivo internacional, pois sua orientação estratégica é para clientes globais e fornecedores globais, $\log o$, as premências de demanda, tecnologia e competição são pautadas pelo contexto internacional (Rugman \& D’Cruz, 1993).

Apresentadas as ideias centrais, este artigo tem como objetivo mostrar que a inovação global em subsidiárias estrangeiras instaladas em mercados emergentes depende dos contextos competitivos nacional e internacional. A inovação global depende diretamente do contexto competitivo internacional. Concomitantemente, a inovação global depende indiretamente do contexto competitivo nacional. Isso porque as subsidiárias precisam se inserir nas redes externas do país hospedeiro para se valer dos benefícios do contexto competitivo nacional. Nesse intuito, o artigo utiliza como objeto de investigação as subsidiárias estrangeiras instaladas no Brasil como representantes das subsidiárias hospedadas em mercados emergentes.

Desse modo, para melhor identificar o processo de inovação global em subsidiárias hospedadas em mercados emergentes (Govindarajan \& Ramamurti, 2011; Govindarajan \& Trimble, 2012), o artigo se propõe a interpretar o papel de um dos elementos dessa inovação, que é o contexto competitivo - nesse caso, o internacional e o doméstico. Ao questionar o impacto do contexto competitivo nacional e internacional, o artigo retorna aos textos de Porter (1990) e Rugman e D'Cruz (1993), discutindo sua aplicação para subsidiárias em mercados emergentes.

O artigo está estruturado na seguinte ordem: além desta introdução, na segunda seção serão apresentados os referenciais teóricos; na seção seguinte, serão apresentados a metodologia de pesquisa, os constructos, a técnica estatística e os índices de ajuste do modelo de equações estruturais; na quarta seção, serão apresentados os resultados e sua discussão; e na última seção, as considerações finais. 


\section{Referencial Teórico}

Nas duas últimas décadas do século XX e na primeira década do século XXI, a reestruturação global da indústria, condicionada em grande parte pela lógica de cadeia de valor produtiva global empregada pelas multinacionais (Hiratuka, 2010), tem aumentado o papel estratégico das subsidiárias estrangeiras. A matriz passou a descentralizar para as subsidiárias o mandato da produção de determinadas linhas de produtos e atividades organizacionais (Birkinshaw, 2001). Essa distribuição das atividades ao redor do mundo não se restringe a atividades de baixa tecnologia, mas também envolve a descentralização da inovação de produtos e processos orientada para a acumulação de recursos e conhecimentos globais (Le Bas \& Sierra, 2002). É uma estratégia produtiva global das multinacionais que culminou numa estrutura em rede (Bartlett \& Ghoshal, 1998), caracterizada pela presença de funções de alta tecnologia fora da matriz (Frost, 1998), inclusive em países em desenvolvimento (Hiratuka, 2005, 2010).

Assim, a vantagem competitiva de que muitas multinacionais desfrutam não se origina exclusivamente a partir de sua matriz, mas pode resultar da articulação e da mobilização de inovações desenvolvidas por filiais (Birkinshaw et al., 1998; Cantwell \& Mudambi, 2005; Foss \& Pedersen, 2002). Logo, as empresas multinacionais têm sido incentivadas a repensar suas estratégias de inovação, visando criar, transferir e explorar o conhecimento disperso ao redor do mundo (Teigland, Fey, \& Birkinshaw, 2000).

Dessa forma, o principal desafio está em conciliar as necessidades de inovação local com as demandas de inovação global (Andersson \& Forsgren, 2006; Teigland et al., 2000). A inovação local é aquela que a subsidiária desenvolve para atender especificamente às necessidades do mercado em que atua. Ela é muito peculiar para o contexto em que foi desenvolvida, e, como não existe nenhum esforço para adaptá-la e alinhá-la aos processos e estratégias globais da multinacional, essa inovação é de difícil transferência para a matriz ou para outra subsidiária. Por sua vez, a inovação global pode, num primeiro momento, ser desenvolvida para atender ao mercado de atuação da subsidiária, mas, num segundo momento, existe todo um esforço da subsidiária para adequar a inovação aos processos e estratégias das outras unidades da multinacional (Birkinshaw, 1997; Rugman \& Verbeke, 2001). A inovação global, também, pode decorrer da demanda interna da multinacional. A matriz aloca recursos e responsabilidades para que uma subsidiária seja a responsável por alguma função da cadeia de valor, e, consequentemente, pelas inovações que pautam a atuação dessa função em escala mundial (Birkinshaw, 1997; Rugman \& Verbeke, 2001). Logo, a inovação global é possível de ser transferida para a matriz e outras subsidiárias, e, como elas têm um alcance global, podem ser usadas por toda a multinacional como fonte de vantagem competitiva (Andersson \& Forsgren, 2006; Moore, 2001; Rugman \& Verbeke, 2001).

Até a década de oitenta do século XX, a inovação global em multinacionais procedeu dos países industrializados para países em desenvolvimento e, numa ação inovadora, em geral unilateral, da matriz dessas multinacionais para suas subsidiárias. No entanto, a crescente internacionalização das empresas, e o consequente aumento na descentralização de P\&D (Frost, 1998) e outras funções (Andersson \& Forsgren, 2006; Frost, Birkinshaw, \& Ensign, 2002; Moore, 2001; Rugman \& Verbeke, 2001) mudaram esta situação. Percebe-se que, a partir da última década do século XX, a transferência de inovação ocorre em várias direções contrárias aos sentidos tradicionais, podendo ocorrer de países em desenvolvimento para países desenvolvidos e das subsidiárias para a matriz. Esse fenômeno tem encontrado apoio em discussões de subsidiárias de empresas multinacionais nos países desenvolvidos (Hakanson \& Nobel, 2000, 2001; Minbaeva, 2008; Minbaeva, Pedersen, Bjorkman, Fey, \& Park, 2003; Yang, Mudambi, \& Meyer, 2008) e em subsidiárias localizadas em mercados emergentes (Govindarajan \& Ramamurti, 2011; Govindarajan \& Trimble, 2012) como o Brasil (Boehe, 2008, 2010; Figueiredo, 2011; Figueiredo \& Brito, 2011; Oliveira, Boehe, \& Borini, 2009). 
Assim, nas páginas a seguir, o artigo analisa um aspecto específico que influencia a inovação global em subsidiárias estrangeiras, a saber, o contexto competitivo aliado à inserção nas redes externas.

As multinacionais estão inseridas em várias redes (intraorganizacionais e interorganizacionais), transformando-se numa teia de redes múltiplas (Dyer \& Hatch, 2006). Do ponto de vista da subsidiária, Andersson, Forsgren e Holm (2002) e Aharoni e Brock (2010) revelam que estas podem ganhar acesso a novos recursos a partir do estreitamento dos laços com as empresas pertencentes à rede externa. Isto porque os relacionamentos se caracterizam como importantes fontes de vantagens competitivas (Forsgren, Holm, \& Johanson, 2005; Johanson \& Vahlne, 2009), tal qual nos estudos de Lakshman e Parente (2008) e Shaohua, Devi e Donald (2007) para o desenvolvimento de produtos.

Entretanto, a inserção em redes não significa uma simples relação entre fornecedor e comprador (Capaldo, 2007; Fritsch \& Kauffeld-Monz, 2008; Granovetter, 1983). Andersson et al. (2002) frisam que as subsidiárias se beneficiam da inserção na rede externa quando o relacionamento é contínuo, de longa duração e caracterizado por uma forte adaptação de comportamentos de ambas as partes (rotinas de negócios, sistemas de planejamento, informações etc.). O resultado é uma relação baseada na confiança mútua. Em outras palavras, trata-se de um relacionamento com elevado enraizamento (embeddedness) entre os parceiros da rede (Forsgren et al., 2005). Esse enraizamento é refletido de duas maneiras. De um lado, pode ser caracterizado como uma forte adaptação do comportamento comercial dos parceiros e uma igualmente forte troca de informações a respeito do mercado. Trata-se das redes de negócios (Andersson et al., 2002). Por outro lado, o enraizamento pode consistir na forte interdependência entre as empresas em termos de produto e desenvolvimento de processos de produção e, consequentemente, uma alta interdependência em termos tecnológicos. Nesse outro caso, trata-se das redes técnicas (Andersson et al., 2002).

Portanto, pressupõe-se que a inovação global estaria associada com as subsidiárias que conseguem se inserir em redes técnicas e de negócios nos mercados emergentes hospedeiros. Esses relacionamentos permitiriam a troca de informações comerciais, de mercado e tecnologias essenciais para o desenvolvimento de inovações no mercado estrangeiro (Shaohua, Devi, \& Donald, 2007). Algumas dessas inovações seriam exclusivamente locais para atender às necessidades e às exigências de cada mercado hospedeiro. Entretanto, devido à particularidade dos recursos e às operações das empresas parceiras e do papel estratégico exercido pela subsidiária, algumas inovações teriam potencial para ser exploradas em outros mercados em que a multinacional atua. Como exemplo disso, devido à escassez de recursos e baixa intensidade tecnológica presente em algum mercado emergente, poderia ser desenvolvido um processo produtivo mais enxuto e com menor custo, porém com igual eficiência que o desenvolvido em outras unidades (Eyring et al., 2011; Govindarajan \& Ramamurti, 2011; Sarti \& Hiratuka, 2011).

Assim, essa inovação desenvolvida em um mercado emergente poderia ser globalizada para as demais subsidiárias da multinacional como meio de reduzir o custo das operações mundiais.

\section{Desse modo, propõe-se que inovações globais estão associadas com a inserção das subsidiárias nas redes técnicas e de negócios do mercado emergente.}

Uma vez discutida a importância da rede externa na promoção da inovação em subsidiárias estrangeiras, a questão que permanece é sobre quando as empresas procuram se enraizar nessas redes. Apesar dos benefícios de participar da rede, a inserção e os relacionamentos com os parceiros são complexos, custosos e tomam tempo (Andersson \& Forsgren, 2006). Desse modo, nem sempre é a primeira alternativa, dado o fato de que as condições do contexto competitivo podem proporcionar condições equiparáveis para a inovação global (Porter, 1990).

Segundo Porter (1990), existiria maior possibilidade de inovação para aquelas empresas inseridas em contextos competitivos caracterizados por elevada competitividade, demanda aquecida e exigente, fornecedores qualificados mundialmente e adequadas condições de infraestrutura física e humana para os negócios. Estudos em subsidiárias estrangeiras de países desenvolvidos (Doz, Santos, 
\& Williamson, 2001; Frost, 2001; Frost et al., 2002), cujo foco era a influência do contexto competitivo sobre as subsidiárias, mostram que as subsidiárias estrangeiras, quando instaladas nessas regiões, também são beneficiadas pelos atributos positivos do ambiente e usam-nos como alavanca para inovações globais. Logo, contextos competitivos dinâmicos e estruturados favorecem a inovação global.

Entretanto, os estudos em questão se referem, em sua grande maioria, às localidades em países desenvolvidos. Por sua vez, quando são analisados os mercados emergentes, é reduzida a probabilidade de encontrar localidades com um contexto competitivo dinâmico e estruturado. Não que sejam inexistentes, porém o principal entrave são as condições da infraestrutura física e humana para fazer negócios. A elevada turbulência do ambiente (Sull \& Escobari, 2005) e as falhas institucionais (Khanna et al., 2010) revelam essas mazelas nos mercados emergentes. Assim, para as empresas estrangeiras, torna-se difícil estabelecer seus negócios sem o apoio de parceiros da rede externa que já aprenderam a operar sob essas condições.

No caso do Brasil, Oliveira, Boehe e Borini (2009) mostram que as subsidiárias avaliam de maneira positiva a condição competitiva, a demanda e a exigência dos consumidores, bem como a qualidade dos fornecedores. Entretanto, o principal entrave está nas condições de infraestrutura. Quando comparado a países desenvolvidos, o país figura como um mercado extremamente burocrático e com regras pouco estáveis e pouco esclarecidas. Isso, por sua vez, pressiona as subsidiárias estrangeiras a estabelecer parcerias estratégicas com os atores da rede externa, tanto para compreender o funcionamento do mercado, como para superar entraves indecifráveis para negócios não nascidos no país. Assim, as subsidiárias estrangeiras dependem da inserção nas redes externas, primeiro como meio de sobrevivência e posteriormente, como meio de aprendizagem das regras do jogo para executar as inovações nos mercados emergentes (Khanna et al., 2010). Devido à particularidade do mercado, a não inserção na rede inviabiliza a aprendizagem para a operação no local, e também as estratégias de inovação global da subsidiária.

Desse modo, fica claro que, quando as subsidiárias estrangeiras pretendem usufruir das condições do contexto competitivo nacional (demanda exigente e aquecida, fornecedores qualificados e rivalidade competitiva) em mercados emergentes, elas procuram aproveitar essas condições para inovação não por meio direto, mas por meio da inserção na rede externa. Dessa forma, explica-se por que a inovação está diretamente associada com a inserção das subsidiárias em redes externas e indiretamente às condições do contexto competitivo.

Por outro lado, é importante ressaltar que as subsidiárias estrangeiras não dependem exclusivamente do contexto competitivo nacional. Embora o modelo de Porter (1990) seja amplamente reconhecido por explicar a competitividade nacional, não é suficiente para abarcar todas as relações competitivas vivenciadas pelas multinacionais (Rugman \& D'Cruz, 1993). Moon, Rugman e Verbeke (1998), ao comparar os contextos competitivos nacionais e internacionais da Coreia do Sul e de Singapura, constataram que a Coreia do Sul possui um contexto competitivo nacional mais robusto que o de Singapura, que, em contrapartida, possui um contexto competitivo internacional melhor que o dos sul-coreanos. Com base na economia de ambos os países, os autores concluíram que tanto os determinantes nacionais quanto os internacionais são importantes para a competitividade. Em conformidade com esses estudos, uma multinacional deve basear-se não somente nas condições do contexto competitivo nacional, mas também no contexto internacional.

Rugman e D'Cruz (1993) argumentam, ainda, que o modelo do contexto nacional é principalmente destinado a explicar as fontes de vantagem competitiva nacional em poder das economias de países avançados, mas é limitado em explicar os níveis e mudanças dinâmicas das economias nos países emergentes, como México e Brasil. Em especial, ressalta-se o caso das multinacionais que atuam como subsidiárias plataformas globais (Oliveira et al., 2009). Essas subsidiárias operam como montadoras e exportadoras de produtos para outros países, usando o país emergente como uma fonte de vantagem comparativa no que tange a mão de obra, o acesso a recursos naturais e a exploração de mercado. As estratégias de inovação global empreendidas por essas subsidiárias são regidas pelos seus clientes, concorrentes e fornecedores globais. Elas usam o país 
como um hub para a montagem dos produtos, que são, em sua grande parte, feitos para exportação. Consequentemente, o que rege os pedidos não são a demanda e a exigência do consumo local, mas sim as do consumidor internacional. Da mesma forma, os fornecedores nem sempre estão no mercado nacional, já que a montadora está integrada a uma cadeia produtiva global. Por sua vez, quanto à competição, ela não é estabelecida com as empresas no mercado hospedeiro, mas com as multinacionais no mercado global.

Quando as relações da cadeia produtiva global se concentram majoritariamente em países desenvolvidos, as subsidiárias ficam expostas a contextos competitivos muito mais dinâmicos e estruturados que o contexto nacional do mercado emergente. Primeiro, a dinâmica competitiva é mais acirrada devido ao elevado grau de competitividade dos fornecedores, concorrentes e compradores. Segundo, a estruturação desse mercado permite que a subsidiária tenha acesso mais fácil aos recursos do ambiente internacional quando comparado ao acesso restrito que precisaria ser intermediado por redes externas no contexto nacional. Consequentemente, é de se esperar que subsidiárias que estabelecem relações produtivas globais e, portanto, são influenciadas pelo contexto competitivo internacional, possam se valer diretamente dos benefícios desse contexto para promover suas estratégias de inovação global (Birkinshaw et al., 1998; Moon et al., 1998). Uma vez apresentadas as ideias centrais, a seguir apresentamos as hipóteses do artigo.

H1a: Inovações globais estão associadas com a inserção das subsidiárias nas redes de negócios do mercado emergente.

H1b: Inovações globais estão associadas com a inserção das subsidiárias nas redes técnicas do mercado emergente.

H2a: A inserção das subsidiárias estrangeiras em redes de negócios está associada com condições satisfatórias do contexto competitivo nacional em mercados emergentes.

H2b: A inserção das subsidiárias estrangeiras em redes técnicas está associada com condições satisfatórias do contexto competitivo nacional em mercados emergentes.

H3: Inovações globais de subsidiárias estrangeiras em mercados emergentes estão associadas com condições satisfatórias de contextos competitivos internacionais de países desenvolvidos.

\section{Metodologia}

Os dados do estudo em questão foram coletados por meio de um survey aplicado on-line com acompanhamento telefônico e destinado para as mil maiores subsidiárias estrangeiras no Brasil, em termos de faturamento. $\mathrm{O}$ respondente era o principal executivo da subsidiária ou algum membro da diretoria designado por esse executivo. Retornaram cento e oitenta e um questionários, dos quais nove foram excluídos por preenchimento incompleto ou errado. Desse modo, a amostra da pesquisa é de cento e setenta e duas empresas, um percentual de $17 \%$ de respostas. A origem das subsidiárias é América do Norte, Europa Ocidental e Japão.

Para a análise de dados, utilizou-se como técnica estatística a modelagem por equações estruturais (SEM). A escolha dessa técnica deve-se ao fato de que o modelo proposto envolve uma relação de caminho (ver Figura 1) entre o contexto competitivo e a inovação global, intermediada pelas redes técnica e de negócios. Adotar uma técnica de regressão linear múltipla não seria o mais adequado, pois mediria isoladamente o impacto do contexto nas redes e das redes na inovação global, sem, contudo, a possibilidade de medir o efeito integrado entre contexto competitivo, redes e inovação global.

Todas as variáveis estão medidas numa escala de Likert de cinco pontos, tendo-se num extremo o valor $\mathbf{1}$ indicando discordo totalmente e, no outro extremo, o valor $\mathbf{5}$, concordo totalmente. Devido à 
impossibilidade de uma medida direta, o construto latente é representado por uma ou mais variáveis latentes, que se constituem nas variáveis observáveis (Hair, Black, Babin, Anderson, \& Tatham, 2009; Kline, 2005). O modelo apresentado neste estudo caracteriza-se como sendo reflexivo, uma vez que a direção de causalidade vai do construto para seus indicadores, conforme a Figura 1. Em outras palavras, nesse tipo de modelo, mudanças no construto causam mudanças nos itens (Jarvis, Mackenzie, \& Podsakoff, 2003).

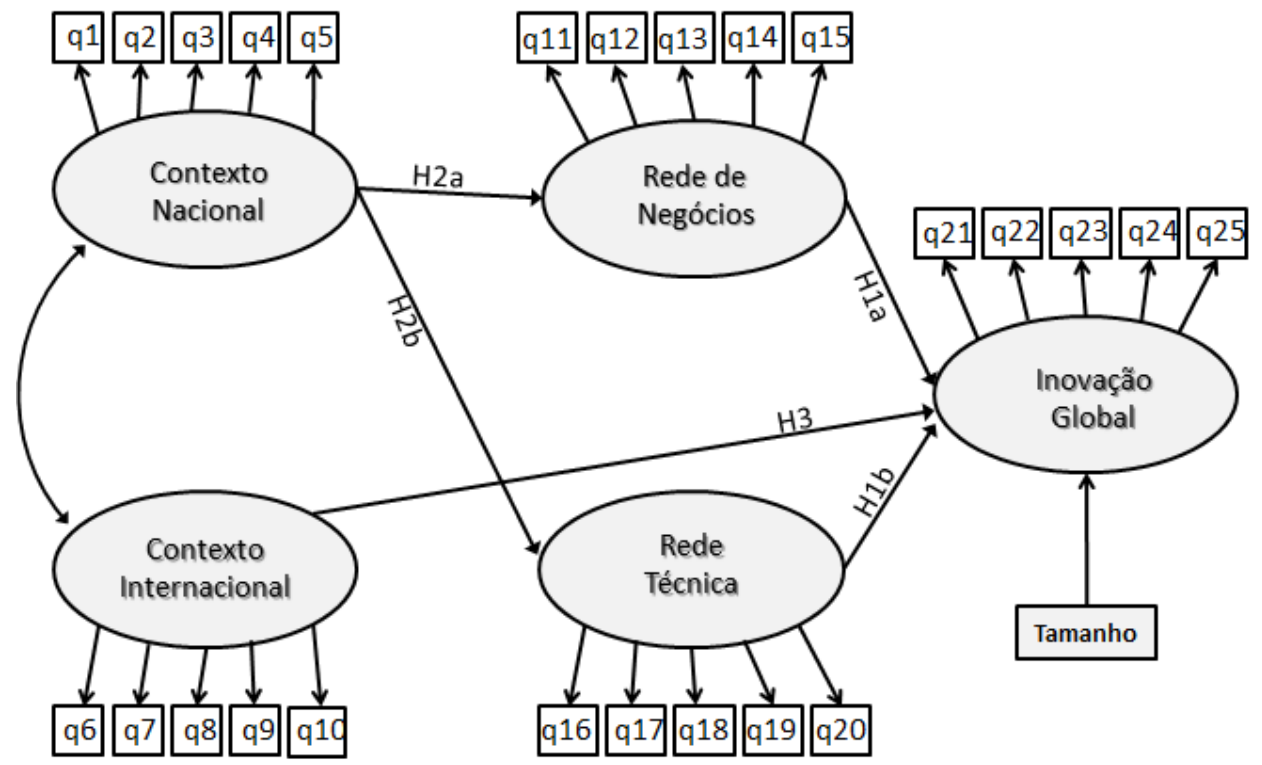

Figura 1. Modelo de Equações Estruturais Proposto.

Fonte: Autores.

A Figura 1 apresenta o modelo resultante das hipóteses postuladas, e as siglas de q1 a q25 representam as variáveis de cada construto. A seguir são apresentados os cinco construtos: contexto nacional, contexto internacional, redes de negócios, rede técnica e inovação global.

O construto do contexto nacional é baseado em Porter (1990) e utilizado por Birkinshaw, Hood e Jonsson (1998) e Borini e Fleury (2010) para verificar como o contexto competitivo influencia as atividades das subsidiárias. As questões são: (q1) tamanho da demanda de mercado no Brasil; (q2) exigência dos consumidores brasileiros; (q3) intensidade da competição no Brasil; (q4) velocidade na inovação de competidores brasileiros; (q5) qualidade dos fornecedores e parceiros brasileiros.

O construto do contexto competitivo internacional (Moon et al., 1998; Rugman \& D'Cruz, 1993) é formado pelas mesmas variáveis do contexto nacional, mas aplicado ao contexto de países desenvolvidos com os quais as subsidiárias estabelecem uma cadeia produtiva global ou uma cadeia de prestação de serviços globais. Assim, mede a influência do(a): (q6) tamanho da demanda do país da matriz que a subsidiária atende; (q7) exigência dos consumidores do país da matriz que a subsidiária atende; (q8) rivalidade dos competidores do país que a subsidiária atende; (q9) velocidade da inovação dos competidores do país da matriz que a subsidiária atende; (q10) qualidade dos fornecedores e parceiros do país da matriz que a subsidiária atende.

O construto de redes de negócios (Lakshman \& Parente, 2008) é formado pelas seguintes variáveis: (q11) nosso relacionamento com nossos principais fornecedores é caracterizado por ser uma relação de longa duração; (q12) nosso relacionamento com nossos principais fornecedores é caracterizado por um alto nível de confiança mútua; (q13) nossos principais fornecedores nunca se comprometem a fazer coisas sem que venham a cumprir o prometido; (q14) nós confiamos em nossos fornecedores no sentido de que eles irão entregar conforme o prometido e dentro do prazo determinado; (q15) nós confiamos nos nossos fornecedores no sentido de que eles irão entregar dentro do padrão de qualidade prometido. 
O construto da rede técnica (Lakshman \& Parente, 2008) é formado pelas seguintes variáveis: (q16) Nossos principais fornecedores possuem habilidade para ajustar rapidamente seu cronograma de produção de acordo com a velocidade e o fluxo de nossa linha de produção; (q17) Nossos principais fornecedores possuem habilidade para ajustar rapidamente seus níveis de estoque de acordo com a velocidade e o fluxo de nossa linha de produção; (q18) Nossa subsidiária normalmente trabalha com times multifuncionais, que são compostos por nossos funcionários e pelos funcionários de nossos principais fornecedores; (q19) Nossos principais fornecedores compreendem como funcionam os processos de nossa empresa; (q20) Nossos principais fornecedores têm uma boa estimativa da composição dos custos referentes aos produtos que fabricamos.

Finalmente, há o construto referente à inovação global, adaptado de Birkinshaw et al. (1998) e Frost, Birkinshaw e Ensign (2002). Este construto é formado pelas seguintes variáveis: (q21) a subsidiária tem permissão da matriz para executar projetos de inovação; (q22) desenvolveu produtos que hoje são vendidos por outras subsidiárias; (q23) desenvolveu processos organizacionais que hoje são adotados em outras subsidiárias; (q24) desenvolveu produtos/processos organizacionais em parceria com fornecedores e que hoje são adotados em outras subsidiárias; (q25) desenvolveu produtos/processos organizacionais em parceria com universidades brasileiras e que hoje são adotados em outras subsidiárias.

Como controle, foi usado o tamanho da subsidiária, caracterizado por uma variável dummy que distingue as subsidiárias com menos de duzentos funcionários (78\% da amostra), consideradas como de pequeno porte, segundo o IBGE, na mesma linha de divisão de tamanho da subsidiária usado no estudo de Borini, Oliveira e Chueke (2012). O tamanho é um importante controle, embora exista controvérsia sobre seu efeito na inovação (Rocha, Borini, \& Spers, 2010) e transferência de práticas (Borini, Oliveira, \& Chueke, 2012) em subsidiárias estrangeiras. Por um lado, as subsidiárias de pequeno porte teriam maior flexibilidade para inovar devido à menor burocracia interna. Contudo, suas inovações tendem a ser muito específicas para a demanda do mercado local. Além disso, devido ao pequeno porte e à provável pouca reputação da subsidiária na rede corporativa interna da multinacional, seria demasiadamente grande o esforço para transformar uma inovação local em inovação global. Por outro lado, as subsidiárias de grande porte, em virtude da sua participação representativa nos ativos da empresa, teriam mais recursos e reputação para conduzir processos de inovação que possam ter um alcance global. Entretanto, a abertura para inovação pode ser lenta ou encontrar várias barreiras em virtude da burocracia interna e certa aversão à incerteza, que é característica de empresas de grande porte, quando comparadas com as pequenas empresas. Assim, o uso do tamanho como variável de controle é importante para entender os direcionadores e os entraves para a inovação global.

\section{Pressupostos e índices de ajuste do modelo}

Analisando os coeficientes de correlação de Pearson na Tabela 1, é possível constatar o pressuposto da linearidade dos dados, uma vez que todos os coeficientes têm valores positivos, menores que 0,85 (Kline, 2005) e com significância p $<0,001$. 
Tabela 1

\section{Correlações}

\begin{tabular}{lccccccc}
\hline & Média & Desvio & I & II & III & IV & V \\
\hline I - Contexto Nacional & 3,86 & 0,57 & 1 & & & & \\
II - Contexto Internacional & 3,4 & 0,73 & $0,234^{* *}$ & 1 & & & \\
III - Rede Técnica & 3,5 & 0,57 & $0,311^{* *}$ & 0,106 & 1 & & \\
IV - Rede de Negócios & 3,8 & 0,45 & $0,229^{* *}$ & $0,169^{*}$ & $0,508^{* *}$ & 1 & \\
V - Inovação & 3,1 & 0,7 & $0,181^{*}$ & $0,324^{* *}$ & $0,342^{* *}$ & $0,168^{*}$ & 1 \\
\hline
\end{tabular}

Nota. Fonte: Autores.

$* * \mathrm{P}<0,01 \quad$ e $* \mathrm{P}<0,05$

Os padrões estipulados como ideais para a validação convergente são, segundo Hair, Black, Babin, Anderson e Tatham (2009): Confiança Composta (CC > 0,7) e Análise de Variância Extraída $(\mathrm{AVE}>0,5)$. Após a obtenção dos coeficientes de regressão padronizados, iniciou-se o cálculo da CC e da AVE. A partir da análise das cargas de cada indicador (q1, q2, q3... q25) e respeitando uma integridade mínima do significado do construto, optou-se pela remoção do indicador de menor carga, pois, segundo Hooper, Coughlan e Mullen (2009), é uma boa prática acessar os ajustes individuais de cada construto para determinar qual item é o mais fraco e, então, removê-lo da análise.

Feita a remoção, o modelo foi recalculado e, assim, foram gerados novos coeficientes de regressão padronizados, os quais foram novamente usados para o cálculo da $\mathrm{CC}$ e da AVE, até que fossem satisfeitos os índices estipulados como padrões (CC >0,7 e AVE >0,5). A Tabela 2 apresenta os cálculos da CC e da AVE, antes e depois do tratamento dos dados.

Tabela 2

Validade Convergente

\begin{tabular}{lcccc}
\hline & \multicolumn{2}{c}{ Antes } & \multicolumn{2}{c}{ Depois } \\
\hline Constructos & CC & AVE & CC & AVE \\
\hline Contexto Nacional & 0,69 & 0,32 & 0,70 & 0,5 \\
Contexto Internacional & 0,76 & 0,4 & 0,76 & 0,52 \\
Redes de Negócios & 0,76 & 0,42 & 0,8 & 0,59 \\
Rede Técnica & 0,82 & 0,49 & 0,79 & 0,57 \\
Inovação Global & 0,74 & 0,37 & 0,75 & 0,5 \\
\hline
\end{tabular}

Nota. Fonte: Autores.

Uma vez verificada a validade convergente, faz-se necessário verificar também a validade discriminante, a qual consiste em medir a diferença entre os construtos. Para realizar tal aferição, utilizou-se o cálculo das variâncias compartilhadas (Tabela 3), método sugerido por Fornell e Larcker (1981). Verifica-se que os construtos atendem as especificidades do método proposto por Fornell e Larcker (1981), uma vez que os $\mathrm{R}^{2}$ são inferiores aos valores de AVE (em negrito), demonstrando que a validação discriminante dos cinco construtos é boa. 
Tabela 3

Validade Discriminante

\begin{tabular}{lccccc}
\hline & $\begin{array}{c}\text { Contexto } \\
\text { Nacional }\end{array}$ & $\begin{array}{c}\text { Contexto } \\
\text { Internacional }\end{array}$ & $\begin{array}{c}\text { Redes de } \\
\text { Negócios }\end{array}$ & Rede Técnica & Inovação \\
\hline Contexto Nacional & $\mathbf{0 , 5 0}$ & & & & \\
Contexto Internacional & 0,003 & $\mathbf{0 , 5 2}$ & & & \\
Redes de Negócios & 0,012 & 0,002 & $\mathbf{0 , 5 9}$ & & \\
Rede Técnica & 0,013 & 0,003 & 0,020 & $\mathbf{0 , 5 7}$ & \\
Inovação & 0,010 & 0,052 & 0,002 & 0,022 & $\mathbf{0 , 5 0}$ \\
\hline
\end{tabular}

Nota. Fonte: Autores.

Por fim, o modelo estrutural resultante apresentou os índices de ajustes classificados como de bom ajuste. A saber: $\mathrm{X}^{2} / \mathrm{gl}=1,58 ; \mathrm{CFI}=0,94$; $\mathrm{GFI}=0,91 ; \mathrm{AGFI}=0,87$; TLI $=0,92$ e RMSEA $=$ 0,058. Os resultados atendem as referências adotadas, e o valor do AGFI está abaixo, porém muito próximo do índice estipulado.

A seguir, o modelo estrutural na Tabela 4 apresenta as seguintes medidas para os construtos e as variáveis que os compõem.

Tabela 4

\section{Medidas}

\begin{tabular}{lccccccc}
\hline Construto & Itens & $\boldsymbol{\lambda}(\mathbf{a})$ & Valor t & $\mathbf{R}^{\mathbf{2}}$ & $\mathbf{A}(\mathbf{b})$ & $\mathbf{C . C}(\mathbf{c})$ & $\mathbf{A V E}(\mathbf{d})$ \\
\hline Contexto & Q3 & 0,63 & 3,18 & 0,40 & 0,65 & 0,7 & 0,50 \\
Nacional & Q5 & 0,77 & $-(\mathrm{e})$ & 0,60 & & & \\
Contexto & Q6 & 0,81 & $-(\mathrm{e})$ & 0,63 & 0,75 & 0,76 & 0,52 \\
Internacional & Q7 & 0,76 & 7,16 & 0,58 & & & \\
& Q8 & 0,58 & 6,45 & 0,34 & & & \\
Rede de & Q13 & 0,42 & 5,4 & 0,19 & 0,75 & 0,8 & 0,59 \\
Negócio & Q14 & 0,88 & $-(\mathrm{e})$ & 0,77 & & & \\
& Q15 & 0,91 & 8,52 & 0,82 & & & \\
Rede Técnica & Q16 & 0,82 & $-(\mathrm{e})$ & 0,68 & 0,78 & 0,8 & 0,57 \\
& Q17 & 0,85 & 8,6 & 0,73 & & & \\
& Q19 & 0,55 & 6,84 & 0,31 & & & \\
Inovação & Q22 & 0,62 & $-(\mathrm{e})$ & 0,38 & 0,74 & 0,75 & 0,51 \\
& Q23 & 0,73 & 6,61 & 0,58 & & & \\
& Q24 & 0,76 & 6,58 & 0,53 & & & \\
\hline
\end{tabular}

Nota. (a) carga padronizada do item; (b) alfa de cronbach; (c) confiança composta; (d) variância extraída; (e) item fixado como 1 na escala. Fonte: Autores.

A partir da Tabela 4, é possível verificar o relacionamento entre as variáveis do modelo, bem como seus respectivos pesos sobre cada construto. Os altos valores das cargas padronizadas $(\lambda)$ que constituem os construtos do contexto nacional (Q5), contexto internacional (Q6), rede de negócio (Q14 e Q15), rede técnica (Q16 e Q17) e o construto da inovação (Q23 e Q24) sugerem a importância dessas variáveis para a predição do modelo. A partir dos critérios estabelecidos por Chin (1998), a variância explicada $\left(\mathrm{R}^{2}\right)$ apresentou, na grande maioria dos itens, valor aceitável $(>0.3)$ e substancial para alguns (>0.67), e apenas um item apresentou uma variância considerada fraca $(>0.19)$. 


\section{Resultados}

A Tabela 5 apresenta os testes de significância das hipóteses, e a Figura 2, o modelo estrutural final. Em termos de indicadores, para aceitação das hipóteses, o Critical Ratio (C.R.) deve ser superior a 1,96 para uma significância em nível 0.05 e acima de 2,58 para uma significância em nível 0.01 . Quatro das cinco hipóteses foram suportadas: H1b, H2a, H2b e H3, sendo somente H1a abaixo do valor mínimo do C.R. e, portanto, não suportada. A variável de controle tamanho apresentou um C.R. de 3,04 sendo, portanto, suportada dentro da significância em nível 0.01. Para a amostra investigada, a variável de controle tamanho revelou-se influente, apresentando um impacto de 0,32 (Figura 2) e evidenciando que, quanto maior o porte da subsidiária, maior o desenvolvimento de inovações globais.

Tabela 5

\section{Teste de Hipóteses}

\begin{tabular}{|c|c|c|c|c|c|c|c|}
\hline Hipóteses & \multicolumn{3}{|c|}{ Relações entre os construtos } & $\lambda^{\mathrm{a}}$ & C.R. ${ }^{b}$ & Sig. $(p)^{c}$ & Resultados \\
\hline H1a & Inovação & $\leftarrow$ & Redes de negócios & $-0,06$ & $-0,64$ & 0,52 & $\begin{array}{c}\text { Não } \\
\text { suportada }\end{array}$ \\
\hline $\mathrm{H} 1 \mathrm{~b}$ & Inovação & $\leftarrow$ & Rede técnica & 0,27 & 2,76 & 0,006 & Suportada \\
\hline $\mathrm{H} 2 \mathrm{a}$ & Rede de negócios & $\leftarrow$ & Contexto nacional & 0,27 & 2,37 & 0,019 & Suportada \\
\hline $\mathrm{H} 2 \mathrm{~b}$ & Rede técnica & $\leftarrow$ & Contexto nacional & 0,26 & 2,27 & 0,024 & Suportada \\
\hline $\mathrm{H} 3$ & Inovação & $\leftarrow$ & Contexto internacional & 0,32 & 3,11 & 0,002 & Suportada \\
\hline \multicolumn{8}{|c|}{ Testes de Apoio } \\
\hline & Inovação & $\leftarrow$ & Contexto nacional & 0,11 & 0,97 & 0,332 & \\
\hline & Rede técnica & $\leftarrow$ & Contexto internacional & 0,13 & 1,42 & 0,156 & \\
\hline & Rede de negócios & $\leftarrow$ & Contexto internacional & 0,12 & 1,34 & 0,181 & \\
\hline \multicolumn{8}{|c|}{ Var. de Controle } \\
\hline & Inovação & $\leftarrow$ & Tamanho & 0,25 & 3,04 & 0,002 & \\
\hline
\end{tabular}

Nota. (a): carga padronizada; (b) Critical Ratio; (c) nível de significância. Fonte: Os autores. $\mathrm{p}<0.05$ e $\mathrm{p}<0.01$.

Em relação à hipótese (H1a), a amostra investigada revelou não existir relação entre a rede de negócio e a inovação auferida pela subsidiária. A análise do modelo apresentado na Figura 2 identifica um efeito nulo $(-0,04)$ das redes de negócios sobre a inovação. $O$ resultado pode sugerir que o fato de a relação não ser significativa indicaria que as redes de negócios, embora baseadas na confiança e na longa duração, não sejam tão propícias ao intercâmbio de conhecimento atrelado à inovação global. Por sua vez, em relação à hipótese (H1b), a análise do modelo apresentado na Figura 2 permite notar que o construto da rede técnica é o que mais impacta na inovação $(0,31)$. A inserção das subsidiárias nas redes técnicas propicia um relacionamento em que os parceiros participam ativamente no desenvolvimento e na produção de novos produtos e processos, o que estimula o intercâmbio de expertises e conhecimento. 


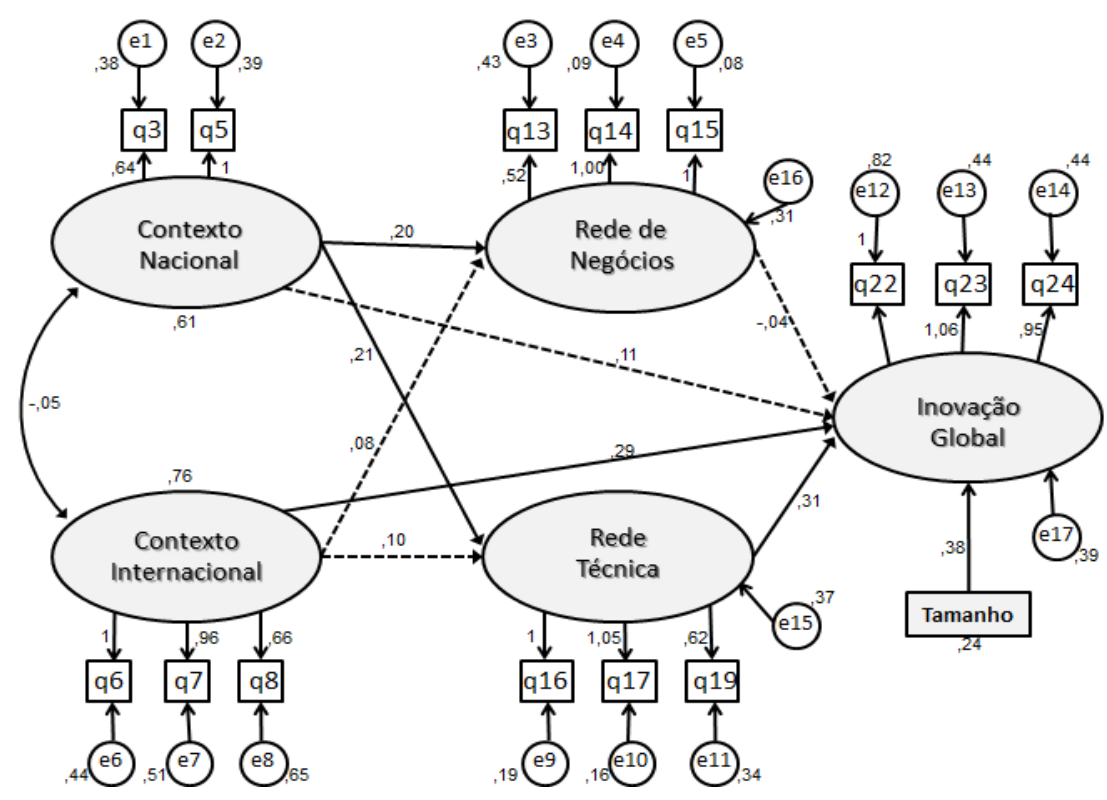

Figura 2. Coeficientes do Modelo Estrutural.

Fonte: Os autores.

Assim, os resultados expressam que a inovação depende de uma efetiva aprendizagem e de uma troca de experiência, tal como constatado por Asmussen, Pedersen e Dhanaraj (2009). Isso quer dizer que, para a inovação global, vale mais as interações produtiva e operacional existentes entre a subsidiária e os parceiros de negócios (rede técnica) do que a interação comercial do relacionamento (rede de negócio).

Muitas vezes, o relacionamento de negócios caracterizado pela confiança mútua e o longo prazo pode existir simplesmente pela conformidade de ambos os lados em prosseguir com os termos estabelecidos, sem que as principais condições iniciais do contrato sejam alteradas. Nesse sentido, esse relacionamento pode até engessar o processo de inovação da empresa. Em virtude da comodidade de se realizarem as transações comerciais como sempre foram realizadas, a subsidiária acaba por não conceder espaço para a mudança, ou o questionamento de pontos críticos, que poderiam ser aperfeiçoados em busca de alguma inovação.

Por sua vez, o resultado mostra que a inovação global emerge da dinâmica do relacionamento técnico, caracterizado pela capacidade da subsidiária de se inserir e construir relacionamentos dinâmicos com os parceiros de negócios. Essa relação, pautada no ajuste contínuo e, por vezes, veloz dos processos produtivos e operacionais, permite que exista uma troca também contínua de experiências e informações entre as partes. Consequentemente, a subsidiária acaba por ter acesso a recursos e a capacidades específicos do local de atuação que isoladamente não teria. Essa relação, como os resultados mostram, acaba por propiciar inovações na subsidiária não só de caráter local, mas também global, ou seja, capazes de serem aproveitadas por outras unidades da corporação multinacional.

Em relação às hipóteses (H2a) e (H2b), a amostra indicou significância tanto na influência exercida do contexto nacional sobre a inserção das subsidiárias em redes de negócios, impacto de $(0,20)$ quanto nas redes técnicas que apresentou impacto de $(0,21)$, conforme o modelo da Figura 2. Por outro lado, é possível verificar na Figura 2 que a relação direta entre o contexto competitivo nacional e a inovação global não é suportada nesse caso das subsidiárias estrangeiras localizadas no Brasil. Isso confirma que o contexto nacional influencia na formação das redes de negócio e das redes técnicas, e, sendo que somente essa última influencia positivamente as inovações globais das subsidiárias, sugere-se que o contexto nacional, por meio das redes técnicas, influencia indiretamente o desenvolvimento de inovações globais das subsidiárias em mercados emergentes. 
Esse resultado é muito interessante e apresenta-se alinhado à evolução da escola nórdica de negócios, quando explica a internacionalização das empresas por meio do investimento direto (Forsgren, 2008). Em suma, concorda com o pressuposto de que o contexto competitivo é importante para a escolha e o comprometimento do investimento direto estrangeiro, porém não de maneira isolada. É essencial que as subsidiárias se insiram nas redes externas para que possam se valer melhor e mais rapidamente dos benefícios das condições do contexto competitivo. Por exemplo, a subsidiária terá maior acesso a fornecedores qualificados não simplesmente por se fazer presente em determinado contexto competitivo nacional, mas somente quando entender a dinâmica comercial, competitiva e produtiva dos fornecedores, o que será viabilizado a partir do momento em que a subsidiária estabelecer relações profícuas com essas empresas.

Essa constatação compartilha e amplia o alcance dos resultados que destacam a prioridade dos relacionamentos interorganizacionais no maior comprometimento estratégico da subsidiária no exterior (Massote, Rezende, \& Versiani, 2010) e na capacidade de desenvolver inovações que possam ser transferidas para outras unidades da multinacional (Shaohua et al., 2007). Ademais, a inserção na rede faz-se necessária para que exista uma aprendizagem efetiva e, assim, possa criar alguma inovação que se diferencie das inovações de outras subsidiárias do grupo (Shaohua et al., 2007). Desse modo, fica evidente que o contexto competitivo nacional é relevante, pois suas peculiaridades permitem que as entidades participantes desenvolvam processos e produtos diferenciados de outros contextos competitivos nacionais. Quando a subsidiária se insere na rede, e consegue aprender com as peculiaridades dos processos e dos produtos desenvolvidos pelas empresas parceiras, ela está apta para desenvolver uma inovação diferenciada dentro da corporação. Basta, então, que a subsidiária consiga fazer essa inovação ter amplitude e reconhecimento global, para que possa ser explorada por outras unidades da corporação.

Em relação à hipótese $\mathrm{H} 3$, verificam-se coeficientes bastante superiores aos encontrados no contexto nacional, em que o coeficiente padronizado corresponde a $(\lambda=0,32), C . R .>3,20$ e, portanto, $\mathrm{p}<0,01$, sendo então H3 suportada. Ademais, a análise do modelo apresentado na Figura 2 identifica um impacto positivo do contexto internacional sobre a criação de inovações $(0,29)$. A aceitação dessa hipótese sugere duas linhas de discussão. Na primeira, como são mais dinâmicos e estruturados, os contextos competitivos de países desenvolvidos influenciam diretamente a criação de inovações globais nas subsidiárias estrangeiras em mercados emergentes. Em outras palavras, para acessar os benefícios do contexto competitivo desses países, as subsidiárias não precisam da intermediação de atores locais em virtude da mais adequada estruturação das instituições do país. Enquanto no Brasil as lacunas institucionais impelem as filiais a exercer parcerias para acessar os benefícios do contexto competitivo nacional, quando essas subsidiárias fazem negócios com atores de países desenvolvidos, têm acesso direto às vantagens do contexto competitivo em razão da presença diminuta de lacunas institucionais. Essa linha de explicação é sustentada pela tese de Khanna, Palepu e Bullock (2010) e Meyer, Estrin, Bhaumik e Peng (2009) acerca das dificuldades e diferenças que as empresas enfrentam ao fazer negócios em economias emergentes.

A segunda linha de discussão que o resultado suscita refere-se à característica da inovação global, a qual é distinta para as subsidiárias que fazem uso do contexto competitivo internacional das que fazem uso do contexto nacional. Essa argumentação encontra respaldo na lógica de cadeia produtiva global, caracterizada pela descentralização das atividades da multinacional (Hiratuka, 2010) e, principalmente, pelos conceitos de iniciativas das subsidiárias exploradas por Birkinshaw (1997). O resultado sugere que as inovações provenientes da relação com o contexto competitivo internacional sejam de dois tipos. Primeiro, inovações demandadas pela matriz ou por outras unidades da corporação multinacional, usando a subsidiária brasileira como elo de uma cadeia global. Tecnicamente, como apontam Zhang, Benedetto e Hoenig (2009), o investimento da matriz nessa atividade de inovação global da subsidiária teria como objetivo reforçar e manter de maneira moderada a capacidade inovadora da unidade para atender a demanda dos clientes globais e a evolução dos fornecedores globais. O segundo tipo de inovação seriam as inovações globais que foram deliberadamente alocadas pela matriz na subsidiária brasileira em virtude de vantagens competitivas ou comparativas que a subsidiária tenha apresentado perante a concorrência interna com outras 
subsidiárias da corporação. Outro aspecto que reforça essa argumentação é a constatação de que as subsidiárias de grande porte realizam mais a inovação global que as de pequeno porte. Além da maior probabilidade dessas grandes empresas atuarem como elo da cadeia de valor global da multinacional, a alocação e a demanda por inovação global tendem a ser maior nas subsidiárias de grande porte, em virtude de sua maior reputação e capacidade de sustentar suas operações no exterior, quando comparadas às de pequeno porte.

Portanto, os resultados sugerem que a inovação global oriunda da relação com o contexto competitivo internacional difere daquela proveniente da relação com o contexto competitivo nacional. Essa última, como já explicitado, decorre de uma iniciativa para responder aos anseios específicos do mercado de operação da subsidiária, e posteriormente é transferida para outras unidades da multinacional; já a primeira está inserida na lógica da cadeia produtiva global, comandada pelas empresas multinacionais.

\section{Conclusão}

O presente artigo identificou que as subsidiárias estrangeiras localizadas em mercados emergentes que praticam atividades de inovação global usam estratégias diferenciadas para acessar os recursos do ambiente externo. Para aproveitar os benefícios do contexto competitivo nacional do país hospedeiro, as subsidiárias precisam se envolver em redes técnicas, como meio de superar as adversidades do local e aprender a operar no mercado emergente. Por sua vez, as subsidiárias integradas em cadeias produtivas globais e que fazem uso de contextos competitivos internacionais localizados em países desenvolvidos não necessitam da intermediação de parceiros para aproveitar os benefícios do contexto competitivo em prol da inovação global.

Este artigo contribui para a literatura de negócios internacionais, mais especificamente sobre a gestão de subsidiárias estrangeiras, em três aspectos. Em primeiro lugar, o resultado do aspecto mediador da inserção da subsidiária na rede técnica como condição para ter acesso aos benefícios do contexto competitivo suporta a proposição de Khanna et al. (2010). Ou seja, as lacunas institucionais do contexto competitivo dos mercados emergentes exigem que as empresas tenham uma estratégia de parcerias com as empresas locais para que possam aprender como fazer negócios no país. Isso sugere que as multinacionais estrangeiras que atuam no Brasil são pressionadas pelo contexto competitivo nacional a conceder maior autonomia para as subsidiárias estabelecerem parcerias locais. Caso contrário, a atuação da empresa no local corre o risco de perder a competitividade por não ter a capacidade de superar as lacunas institucionais.

Um segundo aspecto de contribuição está relacionado à questão das redes. $\mathrm{O}$ artigo confirma a necessidade da inserção em redes, mais especificamente redes técnicas. Essa diferenciação entre a rede técnica e a de negócio ressalta a diferenciação da atuação das subsidiárias em mercados emergentes, uma vez que, para os países desenvolvidos, não existia diferenciação quanto à inserção na rede técnica ou na de negócio (Andersson et al., 2002). Desse modo, fica evidente que, se o objetivo da subsidiária estrangeira é participar de cadeias produtivas globais por meio da inovação, essas empresas devem focar prioritariamente no estabelecimento de redes técnicas; para tanto, os gestores devem concentrar seus esforços em todas as iniciativas de troca de informações e conhecimento com os parceiros locais.

Por fim, um terceiro aspecto de contribuição do artigo refere-se à inovação global. $\mathrm{O}$ artigo confirma a busca das subsidiárias estrangeiras por inovações de alcance global em mercados emergentes (Govindarajan \& Ramamurti, 2011; Govindarajan \& Trimble, 2012), como o Brasil. Contudo, as discussões provenientes dos resultados levantam importante constatação: parecem existir diferentes direcionadores da inovação global. A inovação global pode ser proveniente de um processo de atendimento de necessidades específicas do mercado emergente e que, posteriormente, é transferida para outras unidades, assim como o direcionador da inovação global pode ser a demanda interna da multinacional, que tem a subsidiária como um elo da cadeia de valor global da corporação. Isso quer 
dizer que os gestores com pretensões de ampliar o papel estratégico de suas subsidiárias precisam analisar e avaliar qual das oportunidades de inovação é mais viável para a subsidiária: aquela oriunda do ambiente interno da multinacional, por meio da competição de recursos com outras subsidiárias, ou a decorrente da exploração do ambiente externo do país hospedeiro. Sem dúvida, as duas podem ser viáveis, porém, a administração conjunta das duas possibilidades seria trabalhosa. Parece que o mais adequado seria se valer dos diferentes direcionadores da inovação em momentos distintos da história da subsidiária.

\section{Limitações e sugestões para futuros estudos}

Tais conclusões sugerem futuros estudos que podem avançar em três linhas de investigação. Uma primeira linha seria o aprofundamento da questão do contexto competitivo, para entender melhor o papel das instituições na dinâmica da inovação das subsidiárias estrangeiras no Brasil. A segunda linha está alicerçada à questão das redes, em termos de entender e desvendar de maneira microanalítica os relacionamentos técnicos das subsidiárias com seus parceiros. A terceira linha deveria investigar a natureza e a característica da inovação global, ou seja, desmembrar o conceito de inovação para verificar a estratégia da subsidiária quando a inovação é um imperativo do mercado ou da cadeia de valor da corporação multinacional.

As limitações do presente estudo referem-se principalmente à escolha das variáveis para a composição do modelo de gestão. As variáveis que formam os construtos dos contextos nacional, internacional, redes de negócios, redes técnicas e inovação constituem percepções das próprias subsidiárias a respeito do ambiente. Para evitar o viés social embutido na percepção, poder-se-ia utilizar dados secundários. Na verdade, essa tentativa foi empreendida inicialmente, porém não executada devido à escassez dessas informações para cada setor analisado e para a localidade das 172 empresas. Uma estratégia seria procurar subsidiárias específicas de certas localidades e setores, que tenham os dados secundários disponíveis, e analisar as hipóteses aqui levantadas para verificar se existe a confirmação das mesmas.

Outra limitação está relacionada à técnica estatística utilizada. Grandes amostras são inerentes à SEM; as covariâncias e as correlações são menos estáveis quando estimadas em amostras pequenas, porém, é importante destacar que todos os pressupostos para análise do modelo, como as medidas de ajuste, estão adequados. Dessa forma, salientamos o fato de que uma amostra maior permitirá testar outras relações que não foram apresentadas no artigo, mas de maneira alguma o modelo de SEM aqui apresentado viola os pressupostos para sua utilização.

Por fim, no modelo apresentado não foi investigada a intensidade de internacionalização da multinacional, ou seja, em quantos mercados possui subsidiárias. Essa variável pode ser explorada em futuros estudos, principalmente para explicar a inovação global como demanda da corporação multinacional, em virtude do fato de a empresa ter várias subsidiárias, com diferentes funções da cadeia de valor.

\section{Referências}

Aharoni, Y., \& Brock, D. M. (2010). International business research: looking back and looking forward. Journal of International Management, 16(1), 5-15. doi: 10.1016/j.intman.2009.12.002

Andersson, U., \& Forsgren, M. (2006). Creation and diffusion of competence in MNCs structures, ties and resources at the subsidiary level. In A. Hadjikhani, J. W. Lee, \& J. Johansson (Eds.), Business networks and international marketing (pp. 333-346). Seoul, South Korea: Doo Yang Publishing. 
Andersson, U., Forsgren, M., \& Holm, U. (2002). The strategic impact of external networks: subsidiary performance and competence development in the multinational corporation. Strategic Management Journal, 23(11), 979-996. doi: 10.1002/smj.267

Asmussen, C. G., Pedersen, T., \& Dhanaraj, C. (2009). Host-country environment and subsidiary competence: extending the diamond network model. Journal of International Business Studies, 40(1), 42-57. doi: 10.1057/palgrave.jibs. 8400420

Bartlett, C. A., \& Ghoshal, S. (1998). Transnational management (2a ed.). Boston: McGraw-Hill.

Birkinshaw, J. (1997). Entrepreneurship in multinational corporations: the characteristics of subsidiary initiatives. Strategic Management Journal, 18(3), 207-229. doi: 10.1002/(SICI)10970266(199703)18:3<207::AID-SMJ864>3.0.CO;2-Q

Birkinshaw, J. (2001). Strategy and management in MNE subsidiaries. In A. Rugman \& T. Brewer (Eds.), Oxford handbook of international business (pp. 121-138). Oxford: Oxford University Press.

Birkinshaw, J., Hood, N., \& Jonsson, S. (1998). Building firm-specific advantages in multinational corporations: the role of subsidiary initiative. Strategic Management Journal, 19(3), 221-242. doi: 10.1002/(SICI)1097-0266(199803)19:3<221::AID-SMJ948>3.0.CO;2-P

Boehe, D. M. (2008). Product development in emerging market subsidiaries: the influence of autonomy and internal markets on subsidiary roles. International Journal of Innovation and Technology Management, 5(1), 29-53. doi: 10.1142/S021987700800128X

Boehe, D. M. (2010). Captive offshoring of new product development in brazil: how does arbitrage influence local, collaborative relationships?. Management International Review, 50(6), 747-773. doi: $10.1007 / \mathrm{s} 11575-010-0054-\mathrm{z}$

Borini, F. M., \& Fleury, M. T. L. (2010). O desenvolvimento de competências organizacionais em diferentes modelos gerenciais de subsidiárias de empresas multinacionais brasileiras. Revista de Administração Contemporânea, 14(4), 575-593. Recuperado de http://www.scielo.br/pdf/rac/v14n4/02.pdf. doi: 10.1590/S1415-65552010000400002

Borini, F. M., Oliveira, M. M., Jr., \& Chueke G. V. (2012). Transferência reversa de práticas de responsabilidade social corporativa em subsidiárias estrangeiras no Brasil. BBR. Brazilian Business Review, 9(5), 81-105.

Cantwell, J., \& Mudambi, R. (2005). MNE Competence-creating subsidiary mandates. Strategic Management Journal, 26(12), 1109-1128. doi: 10.1002/smj.497

Capaldo, A. (2007). Network structure and innovation: the leveraging of a dual network as a distinctive relational capability. Strategic Management Journal, 28(6), 585-608. doi: $10.1002 /$ smj. 621

Chin, W. W. (1998). The partial least squares approach to structural equation modeling. In G. A. Marcoulides (Ed.), Modern methods for business research (pp. 295-336). New York: Lawrence Erlbaum Associates.

Doz, Y., Santos, J., \& Williamson, P. (2001). From global to metanational: how companies win in the knowledge economy. Boston: Harvard Business School Press.

Dyer, J., \& Hatch, N. (2006). Relation specific capabilities and barriers to knowledge transfers: creating advantage through network relationships. Marriott: Brigham Young.

Eyring, M., Jonsson, M., \& Nair, N. (2011). New business model in emerging markets. Harvard Business Review, 89(1), 88-95. 
Figueiredo, P. N. (2011). The role of dual embeddedness in the innovative performance of MNE subsidiaries: evidence from Brazil. Journal of Management Studies, 48(2), 417-440. doi: 10.1111/j.1467-6486.2010.00965.x

Figueiredo, P. N., \& Brito, K. (2011). The innovation performance of MNE subsidiaries and local embeddedness: evidence from an emerging economy. Journal of Evolutionary Economics, 21(1), 141-165. doi: 10.1007/s00191-010-0180-6

Fornell, C., \& Larcker, D. F. (1981). Evaluating structural equation models with unobservable variables and measurement error: algebra and statistics. Journal of Marketing, 18(1), 39-59.

Forsgren, M. (2008). Theories of the multinational firm: a multidimensional creature in the global economy. Cheltenham, UK: Edward Elgar.

Forsgren, M., Holm, U., \& Johanson, J. (2005). Managing the embedded multinational: a business network view. Cheltenham: Edward Elgar.

Foss, N., \& Pedersen, T. (2002). Transferring knowledge in MNCs: the role of sources of subsidiary knowledge and organizational context. Journal of International Management, 8(1), 1-19. doi: 10.1016/S1075-4253(01)00054-0

Fritsch, M., \& Kauffeld-Monz, M. (2008). The impact of network structure on knowledge transfer: an application of social network analysis in the context of regional innovation networks. The Annals of Regional Science, 44(1), 21-38. doi: 10.1007/s00168-008-0245-8

Frost, A. (1998). The geographic sources of innovation in the multinational enterprise: US subsidiaries and host country spillovers, 1980-1990 (PhD thesis). Sloan School of Management, Cambidge, MA, MIT.

Frost, T. (2001). The geographic sources of foreign subsidiaries innovations. Strategic Management Journal, 22(2), 101-123. doi: 10.1002/1097-0266(200101)22:2<101::AID-SMJ155>3.0.CO;2-G

Frost, T., Birkinshaw, J., \& Ensign, P. (2002). Centers of excellence in multinational corporations. Strategic Management Journal, 23(11), 997-1018. doi: 10.1002/smj.273

Govindarajan, V., \& Ramamurti, R. (2011). Reverse innovation, emerging markets, and global strategy. Global Strategy Journal, 1(3-4), 191-205. doi: 10.1002/gsj.23

Govindarajan, V., \& Trimble, C. (2012). Reverse innovation. Boston: Harvard Business Press.

Granovetter, M. (1983). The strength of weak ties: a network theory revisited. Sociological Theory, 1(2), 201-233. doi: 10.2307/202051

Hair J. F., Black, W. C., Babin, B. J., Anderson, R. E., \& Tatham, R. L. (2009). Análise multivariada de dados (6a ed.). Porto Alegre: Bookman.

Hakanson, L., \& Nobel, R. (2000). Technology characteristics and reverse technology transfer. Management International Review, 40(1), 29-48.

Hakanson, L., \& Nobel, R. (2001). Organizational characteristics and reverse technology transfer. Management International Review, 41(4), 395-420.

Hiratuka, C. (2005). Internacionalização de atividades de pesquisa e desenvolvimento das empresas transnacionais: análise da inserção das filiais brasileiras. São Paulo em Perspectiva, 19(1), 105114. doi: 10.1590/S0102-88392005000100009 
Hiratuka, C. (2010). A reorganização das empresas transnacionais e sua influência sobre o comércio internacional no período recente. In L. A. Silva \& R. P. Leão (Orgs.), Comércio internacional: aspectos teóricos e as experiências indiana e chinesa (pp. 11-35). Brasília: Ipea.

Hooper, D., Coughlan, J., \& Mullen, M. (2009). Structural equation modeling: guidelines for determining model fit. Electronic Journal of Business Research Methods, 6(1), 53-60.

Jarvis, C. B., Mackenzie, S., \& Podsakoff, P. (2003). A critical review of construct indicators and measurement model misspecification in marketing and consumer research. Journal of Consumer Research, 30(2), 199-218. doi: 10.1086/376806

Johanson, J., \& Vahlne, J. E. (2009). The uppsala internationalization process revisited: from liability of foreigness to liability of outsidership. Journal of International Business Studies, 40(9), 11411431. doi: $10.1057 /$ jibs.2009.24

Khanna, T., Palepu, K., \& Bullock, R. (2010). Winning in emerging markets. Havard Business Review, 88(6), 80-85.

Kline, R. B. (2005). Principles and practice of structural equation modeling (2nd ed.). New York: The Guilford Press.

Lakshman, C., \& Parente, R. C. (2008). Supplier-focused knowledge management in the automobile industry and its implications for product performace. Journal of Management Studies, 45(2), 317-342. doi: 10.1111/j.1467-6486.2007.00743.x

Le Bas, C., \& Sierra, C. (2002). Location versus home country advantages in R\&D activities: some further results on multinationals locational strategies. Research Policy, 31(4), 589-609. doi: $10.1016 / \mathrm{S} 0048-7333(01) 00128-7$

Massote, C. G., Rezende, S. F. L., \& Versiani, A. F. (2010). A dinâmica de relacionamentos nacionais e internacionais em processos de internacionalização: um estudo de caso de uma agência norteamericana de publicidade no mercado Brasileiro. Revista de Administração Contemporânea, 14(1), 61-79. Recuperado de http://www.scielo.br/pdf/rac/v14n1/05.pdf. doi: 10.1590/S141565552010000100005

Meyer, K. E., Estrin, S., Bhaumik, S., \& Peng, M. (2009). Institutions, resources, and entry strategies in emerging economies. Strategic Management Journal, 30(1), 61-80. doi: 10.1002/smj.720

Minbaeva, D. (2008). HRM Practices affecting extrinsic and intrinsic motivation of knowledge receivers and their effect on intra-MNC knowledge transfer. International Business Review, 17(6), 703-713. doi: 10.1016/j.ibusrev.2008.08.001

Minbaeva, D., Pedersen, T., Bjorkman, I., Fey, C., \& Park, H. (2003). MNC Knowledge transfer, subsidiary absorptive capacity and knowledge transfer. Journal of International Business Studies, 34(6), 586-599. doi:10.1057/palgrave.jibs. 8400056

Moon, H. C., Rugman, A. M., \& Verbeke, A. (1998). A generalized double diamond approach to the global competitiveness of Korea and Singapore. International Business Review, 7(2), 135-150. doi: 10.1016/S0969-5931(98)00002-X

Moore, K. (2001). A strategy for subsidiaries: centers of excellence to build subsidiary specific advantages. Management International Review, 41(3), 275-290.

Oliveira, M. M., Jr., Boehe, D. M., \& Borini, F. M. (2009). Estratégia e inovação em corporações multinacionais: a transformação das subsidiárias brasileiras. São Paulo: Saraiva.

Porter, M. (1990). Vantagem competitiva: criando e sustentando um desempenho superior. Rio de Janeiro: Campus. 
Rocha, T. V., Borini, F. M., \& Spers, E. E. (2010). A autonomia de marketing das subsidiárias estrangeiras no Brasil para desenvolvimento de novos produtos em multinacionais. Revista de Administração, 45(4), 328-342.

Rugman, A. M., \& D'Cruz, J. R. (1993). The double diamond model of international competitiveness: the Canadian experience. Management International Review, 33(2), 17-39.

Rugman, A. M., \& Verbeke, A. (2001). Subsidiary specific advantages in multinational enterprises. Strategic Management Journal, 22(3), 237-250. doi: 10.1002/smj.153

Sarti, F., \& Hiratuka, C. (2011). Indústria mundial: mudanças e tendências recentes [Texto para discussão, $\mathrm{N}^{\circ}$ 186]. Campinas, SP: IE-Unicamp.

Shaohua, C. M., Devi, R. G., \& Donald, E. H. (2007). Foreign subsidiaries' learning from local environments: an empirical test. Management International Review, 47(1), 79-102. doi: $10.1007 / \mathrm{s} 11575-007-0005-5$

Sull, D. N., \& Escobari, M. (2005). Success against the odds: what Brazilian champions teach us about thriving in unpredictable markets. London: Elsevier.

Teigland, R., Fey, C., \& Birkinshaw, J. (2000). Knowledge dissemination in global R\&D operations: an empirical study of multinationals in the high technology electronics industry. Management International Review, 40(1), 49-77.

Yang, Q., Mudambi, R., \& Meyer, K. E. (2008). Convention and reverse knowledge flows in multinational corporations. Journal of Management, 34(5), 882-902. doi: $10.1177 / 0149206308321546$

Zhang, J. C., Benedetto, A., \& Hoenig, S. (2009). Product development strategy, product innovation performance, and the mediating role of knowledge utilization: evidence from subsidiaries in China. Journal of International Marketing, 17(2), 42-58. doi: 10.1509/jimk.17.2.42 